\section{$\underset{\text { \& migrations }}{\text { hommes }}$}

\section{Hommes \& migrations}

Revue française de référence sur les dynamiques

migratoires

$1305 \mid 2014$

L'exil chilien en France

\title{
Les exilés politiques chiliens en France, quarante ans après le coup d'État
}

\section{Mabel Verdi Rademacher}

\section{(2) OpenEdition}

\section{Journals}

\section{Édition électronique}

URL : http://journals.openedition.org/hommesmigrations/2715

DOI : 10.4000/hommesmigrations. 2715

ISSN : 2262-3353

\section{Éditeur}

Musée national de l'histoire de l'immigration

\section{Édition imprimée}

Date de publication : 1 janvier 2014

Pagination : 41-47

ISBN : $978-2919-040261$

ISSN : $1142-852 X$

\section{Référence électronique}

Mabel Verdi Rademacher, "Les exilés politiques chiliens en France, quarante ans après le coup d'État », Hommes \& migrations [En ligne], 1305 | 2014, mis en ligne le 01 janvier 2017, consulté le 10 décembre 2020. URL : http://journals.openedition.org/hommesmigrations/2715 ; DOI : https:// doi.org/10.4000/hommesmigrations.2715 


\title{
LES EXILÉS POLITIQUES CHILIENS EN FRANCE QUARANTE ANS APRĖS LE COUP D'ÉTAT
}

par MABEL VERDI RADEMACHER, doctorante en sociologie à l'École des hautes études en sciences sociales (EHESS), Paris.

\author{
II n'y a pas de profil type de l'exilé chilien en France. \\ Au contraire, ils constituent une catégorie hétérogène avec \\ un attachement variable à leur pays d'origine et des modalités \\ différentes de socialisation en France. Ces critères permettent \\ de définir plusieurs groupes parmi eux. Après I'abandon \\ du mythe du retour au Chili, leur installation définitive dans \\ leur pays d'accueil repose sur les liens forts tissés avec \\ leurs compatriotes résidant en France, comme avec I'ensemble \\ de la société française.
}

La problématique des exilés politiques chiliens a été abordée par diverses études qui rendent compte de leur vie de migrants ${ }^{1}$. Ces travaux soulignent que, pendant les premières années d'exil, ils étaient fortement engagés dans la lutte et la résistance contre la dictature de Pinochet. La vie associative était riche à l'époque : en témoigne la création de diverses associations à but politique, mais également sportif et culturel. Le mythe du retour était très présent, les exilés vivaient avec leurs "valises prêtes” pour repartir. L'installation à long terme de la dictature (du 11 septembre 1973 au 11 mars 1990) a modifié leurs projets ; ils ont commencé à s'investir davantage dans leur processus d'intégration aux pays qui les ont accueillis, en accordant moins de place à la vie associative.

Une fois levée l'interdiction de retourner dans leur pays $^{2}$, certains exilés sont repartis au $\mathrm{Chili}^{3}$, mais pour nombre de ces retornados, cette expérience a été douloureuse et difficile ${ }^{4}$ et a souvent abouti à un nouvel exil. À ce propos, certains auteurs s'interrogent sur la formation d'une diaspora chilienne ${ }^{5}$. 
Le retour à la démocratie au Chili a mis un terme à la lutte et au projet commun des exilés. La fin de l'exil et du statut de réfugié ${ }^{6}$ a-t-elle fait disparaître la figure de l'exilé politique et l'autoreconnaissance des personnes qui avaient ce statut? Ces individus sont-ils passés de l'exil à la migration? Aujourd'hui, quarante ans après le coup d'État, quelles sont leurs attaches ? À partir de l'analyse des biographies de mobilité et des réseaux sociaux des migrants chiliens en France ${ }^{7}$, nous souhaitons contribuer à la compréhension du destin des exilés, tout en interrogeant leurs liens et leurs logiques d'appartenance aux deux sociétés dans lesquelles ils s'insèrent.

\section{Les exilés politiques chiliens : un groupe hétérogène}

Les exilés politiques chiliens ne sont pas une catégorie homogène. Leurs différences sont observables selon plusieurs points de vue, parmi lesquels : l'origine sociale (toutes les classes sociales sont concernées), leur activité (des diplômés, des étudiants, des ouvriers), les caractéristiques sociodémographiques (des hommes, des femmes, des adultes, des jeunes, des enfants), la manière dont ils ont quitté le Chili et se sont installés dans le pays d'exil (seuls ou en famille; en tête d'un exil familial ou en tant qu'accompagnant d'un autre exilé ; à travers des réseaux préétablis ou par l'établissement de contacts sur place) ; et le parcours migratoire dans le pays d'exil.

D'après les récits des migrants, il existe une dimension subjective concernant la légitimité de la condition d'exilé, qui diversifie encore plus ce groupe de Chiliens : d'un côté, se trouveraient les "vrais" exilés politiques (ceux qui ont vécu la torture, la prison et l'expulsion du pays), et, de l'autre, ceux qui sont partis en exil de manière plus ou moins délibérée car ils se sentaient en danger et/ou parce qu'ils ont vécu les conséquences économiques de la dictature. Cette hétérogénéité est également présente en ce qui concerne les liens établis après l'exil. Nous avons repéré trois types d'appartenance : ceux qui restent attachés à leur pays d'origine sans manifester d'intérêt particulier pour leur pays de résidence ; ceux qui sont attachés à leur pays d'origine et à leur pays de résidence ; ceux qui sont attachés à leur pays de résidence sans s'intéresser à leur pays d'origine.

\section{Le primat des origines}

Les exilés politiques de la première catégorie se caractérisent par un réseau social constitué principalement de contacts familiaux et amicaux chiliens (entre $73 \%$ et $78 \%$ de leurs contacts sont des compatriotes), dont presque la moitié résident au Chili. Ces exilés font des voyages réguliers dans leur pays d'origine (au moins une fois par an), et entretiennent des liens à distance de manière fréquente avec une diversité de personnes et en utilisant une variété de dispositifs de communication.

Christian Licoppe souligne que les technologies permettent de "négocier un compromis entre le déficit relationnel que crée l'absence et les investissements qu'il faut désormais mobiliser pour entretenir cette 'conversation continuelle et ininterrompue' qui est la matière même du lien ${ }^{8}$. En effet, la sociabilité de ces exilés - bien équipés et très connectés - est fortement médiatisée, et lors des rencontres en coprésence physique (notamment grâce aux voyages au Chili), "il n'y a pratiquement rien de nouveau à se raconter, nous poursuivons nos conversations", affirme Osvaldo (61 ans).

5. Claudio Bolzman, “De l'exil à la diaspora : l'exemple de la migration chilienne”, in Autrepart, n²2, 2002, pp. 91-107; José. del Pozo, “Los chilenos en el exterior: ¿de la emigración y el exilio a la diáspora? El caso de Montreal”, in Revue européenne des migrations internationales, vol. $20, n^{\circ}{ }^{1}$, 2004 ; Nicolas Prognon, “La diaspora chilienne en France. L'exil et le retour (1973-1994)”, thèse université Toulouse-Le Mirail, 2002. 6. Le $1^{\text {er }}$ septembre 1988 , le décret $n^{\circ} 203$ du ministère de l'Intérieur a mis fin à l'exil. Cependant, les exilés politiques chiliens maintiennent leur statut de réfugiés en France jusqu'en 1994. 7. Cet article se fonde sur les observations effectuées au cours de notre recherche sur la mobilité et les réseaux sociaux des migrants chiliens en France. Le corpus est constitué par les récits de 60 migrants, dont des exilés, des migrants économiques ou professionnels, des migrants venus pour des raisons personnelles, des étudiants qui résident en France depuis au moins quatre ans et des enfants de migrants chiliens nés en France ou arrivés pendant l'enfance. Les données ont été recueillies principalement en Île-de-France entre 2009 et 2011. 8. Christian Licoppe, “Sociabilité et technologies de communication : deux modalités d'entretien de liens interpersonnels dans le contexte du dépliement des dispositifs de communications”, in Réseaux n¹12-113, 2002, pp. 173-210. 


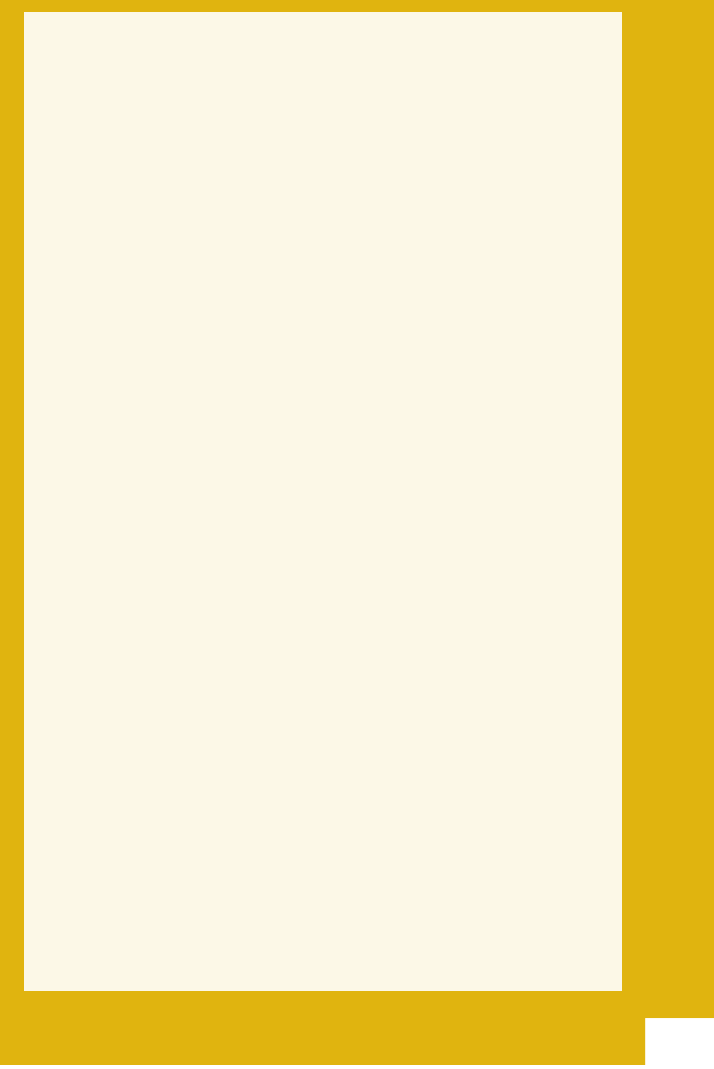

Revue Araucaria de Chile (c) D.R.

Pour ces exilés politiques, la moitié de leurs liens résident en France. Mais il s'agit essentiellement d'amitiés et de connaissances d'origine chilienne, dont toutes n'ont pas été rencontrées dans le cadre de l'exil. Ces exilés n'ont pas de liens familiaux en France, soit parce qu'ils sont célibataires et que leur famille ascendante réside au Chili, soit parce qu'ils sont divorcés et que leur descendance habite dans des pays autres que la France, en constituant parfois une famille transnationale. Dans ces cas, des bifurcations biographiques - notamment des séparations ou des déménagements vers une autre ville - ou l'instabilité des parcours socioprofessionnels ont eu comme conséquence la perte de plusieurs liens sur place. Tout cela se traduit par un attachement plus faible à la France.

Même s'ils continuent à se définir comme "de gauche", ces exilés questionnent la légitimité de leur statut de réfugié, car ils sentent qu'ils ne sont pas de "vrais" exilés politiques. Ce sentiment les a conduits dès le début de leur séjour à prendre de la distance par rapport aux autres exilés et à adhérer peu ou pas du tout à des associations chiliennes, politiques ou non. De nos jours, ils ne se sentent pas concernés par les manifestations de soutien au Chili lorsque ce pays traverse des difficultés particulières. Cet "isolement" vis-à-vis des autres Chiliens résidant en France peut s'expliquer par le statut ambivalent de ces individus, ressenti par eux-mêmes et questionné par la communauté des exilés.

D'autre part, leur position par rapport à la France est également ambivalente. Étant donnéleur sentiment d' 'illégitimité" en ce qui concerne leur statut, ils n'ont pas voulu ou ils n'ont pas pu profiter, en début de séjour, de certains bénéfices accordés par ce pays, ni du capital social des exilés fondé sur leur dimension collective. Ainsi, la déqualification professionnelle, qu'ils ont fortement vécue, a été surmontée à travers des stratégies plus individuelles. Pourtant, le bilan après tant d'années reste négatif : ils ressentent une discrimination de la part de la société française, comme d'ailleurs beaucoup d'autres migrants. Ils sont critiques envers ce pays également sur le plan des relations humaines - un domaine où la société chilienne leur paraît supérieure en comparaison. S'ils restent en France, c'est principalement en raison de leur carrière professionnelle, mais, à l'approche de la retraite, un questionnement s'ouvre sur leur avenir. Le mythe du retour est ainsi très présent.

Pour ces exilés politiques, l'attachement à leur pays d'origine se centre principalement sur les liens sociaux, car ils ne manifestent pas d'intérêt parti- 
culier pour participer à un projet de pays. On pourrait même dire que leur retour n'est pas un retour "idéologique", mais "nostalgique".

\section{Dans l'entre-deux}

La plupart des exilés politiques interviewés possèdent un double attachement. Dans ce cas, leur réseau social est constitué majoritairement de contacts chiliens (entre $68 \%$ et $91 \%$ de leurs contacts). Nonobstant, ce groupe se distingue du précédent par un plus grand nombre de contacts chiliens en France qu'au Chili. Tous ces exilés se sont déplacés avec leur famille ou ont formé une famille nucléaire en France, ce qui explique en partie le grand nombre de contacts chiliens qu'ils ont dans ce pays. Cependant, si Outre le partage les liens familiaux sont bien d'un événement passé, un facteur qui favorise les liens entre les Chiliens en France

est la participation à des

groupes réunissant plusieurs ressortissants, tels que les associations de Chiliens. représentés, la plupart de ces contacts correspondent à des liens d'amitié. En règle générale, on sait que la sociabilité des jeunes se caractérise par la présence prédominante des liens d'amitié, tandis que celle des adultes se caractérise par la prédominance des liens familiaux ${ }^{10}$. Or, chez ces exilés chiliens, la sociabilité des adultes est proche de celle des jeunes. Le primat des relations amicales sur les relations familiales peut se comprendre par le fait qu'à l'étranger se produisent souvent une intensification des liens et un élargissement du terme "ami".

Certains de ces liens sont tels que, d'une certaine manière, ils complètent et parfois même remplacent les liens entretenus avec la famille résidant dans le pays d'origine. Nous observons ainsi la formation d'une "quasi-famille", le terme "quasi" désignant la non-appartenance naturelle. Il ne s'agit pas de liens de parenté biologique, mais ils peuvent être considérés comme tels en raison de leur fonction sociale et par les fonctions de solidarité et de partage, qui sont normalement assumées par la famille. Les récits des exilés révèlent que la relation va au-delà de l'amitié, ces nouveaux contacts étant désignés par des termes propres aux liens de parenté (frères, sœurs, cousins, oncles, etc.).

Bien que l'on puisse retrouver ce phénomène de quasi-famille chez beaucoup de migrants, il semble se manifester avec plus de force chez les exilés politiques qui ont noué leurs relations d'amitié dans l'expérience commune de l'exil. En effet, tous ces migrants se considèrent comme de "vrais" exilés politiques.

Outre le partage d'un événement passé, un facteur qui favorise les liens entre les Chiliens en France est la participation à des groupes réunissant plusieurs ressortissants, tels que les associations de Chiliens. En effet, plusieurs de ces exilés ont une vie associative active, notamment au sein d'organisations à vocation politique, mais également dans des groupes sportifs et folkloriques. Dans la plupart des cas, le "monde affectif" se construit autour de cette participation qui leur permet d'être en contact avec d'autres Chiliens, en dehors des liens familiaux ${ }^{11}$.

Nous observons que très souvent ces exilés ont des réseaux contextualisés, c'est-à-dire qui montrent "une relative dépendance des relations par rapport au contexte social dans lequel elles s'inscrivent ${ }^{12 "}$. Cependant, on trouve aussi des personnes qui se sont éloignées du militantisme et de la vie associative chilienne, mais qui gardent des liens d'amitié avec leurs ex-camarades de lutte. Parfois, elles ont aussi créé ou réactivé des liens forts avec des Chiliens résidant au Chili.

Concernant la relation de ces exilés politiques avec leur pays d'origine, leurs voyages ne sont pas réguliers mais plutôt contingents. D'autre part, l'entretien des liens à distance avec leurs contacts résidant au Chili - qui sont pour la plupart des membres de 
leur famille - s'effectue de manière occasionnelle. Nonobstant, la communication reste satisfaisante à leurs yeux et leurs contacts au Chili continuent d'être importants pour eux.

Ces exilés politiques montrent aussi leur attachement à leur pays d'origine en participant, en France, à des événements liés au Chili. Fanny Jedlicki ${ }^{13}$ observe que l'arrestation de Pinochet à Londres en $1998^{14}$ réactive la mémoire collective de l'exil. Les personnes interviewées évoquent cet événement comme le dernier qui ait permis de mobiliser de manière massive les Chiliens exilés résidant dans différents pays d'Europe.

Au début de leur exil, les Chiliens étaient le groupe le plus important parmi les communautés de Latino-Américains en France. Or l'importance de la France pour les exilés politiques chiliens a été quantitative et qualitative. La chute de l'Unité populaire et du président Salvador Allende a mobilisé les partis politiques et les syndicats de gauche qui ont créé des "comités de solidarité" pour sensibiliser la population française. À l'époque, la vie associative des exilés était très intense. Cependant, la longue durée de la dictature a affaibli les luttes, le soutien des Français a diminué progressivement et les exilés se sont investis de manière prioritaire dans leur intégration en France, en accordant moins d'importance à l'activisme politique.

La dynamique actuelle de la vie communautaire des exilés dépend des circonstances. Même si des associations de Chiliens continuent d'exister, elles se caractérisent aujourd'hui par la poursuite d'objectifs très concrets et contextuels. Les luttes politiques d'autrefois n'existent plus. Les exilés continuent donc à être actifs, mais leurs revendications et leurs mobilisations rejoignent souvent celles d'autres migrants chiliens non liés à l'exil politique. Aux yeux de ces exilés politiques, l'attachement au Chili n'est pas incompatible avec leur implication en France, pays auquel ils sont habitués, au point d'abandonner le mythe du retour. Cela s'explique

par le fait que, reconnaissants de l'accueil bienveillant de la France, ces exilés ont passé plus de la moitié de leur vie dans ce pays, s'y sont intégrés, y ont créé des liens forts, et ont eu une descendance qui souhaite rester. En outre, ils sont conscients de la difficulté de se réinsérer au Chili.

\section{L'adoption d'un pays}

Le réseau des exilés politiques chiliens attachés davantage à la France qu'à leur pays d'origine est constitué notamment par des contacts chiliens et français. À la différence des cas précédents, pour ces exilés, la proportion des liens chiliens est moins significative (environ la moitié de leurs contacts). En outre, ces exilés ont en partage le fait d'être en couples binationaux, ce qui explique en partie la diversité de leurs contacts.

Pour ces exilés, les liens avec des Chiliens résidant au Chili sont rares (entre $5 \%$ et $15 \%$ ). Leurs familles résident soit en France, soit dans d'autres pays, mais dans tous les cas hors du Chili. Cependant, la perte de liens forts avec leur pays d'origine ne s'explique pas seulement par l'absence de liens familiaux, mais également par la rupture avec leurs anciens cama-

Ils ne vivent pas dans le mythe du retour. Depuis qu'ils ont quitté leur pays, ils ont décidé de commencer une nouvelle vie. rades de lutte. En effet, ces personnes ont une position critique par rapport au Chili et mettent en question les faits politiques et la manière dont la lutte contre la dictature a été menée. Ces exilés politiques ne sont jamais retournés au Chili.

Ayant eu un passé militant actif, ils ne s'intéressent plus à la vie politique et associative et se sont davantage investis dans leur parcours professionnel. Ils ne participent pas non plus aux éventuelles activités de soutien au Chili. Et, même s'ils peuvent continuer à manifester un certain intérêt pour la 
politique, celui-ci repose essentiellement sur la politique française. D'autre part, leurs préoccupations se centrent maintenant sur des domaines comme la religion ou le monde artistique et culturel.

Ces exilés se sont bien intégrés à leur société d'accueil et ont de nombreux contacts français. Leurs contacts chiliens - des membres de leur famille, des amis - sont de provenances diverses et ne sont pas seulement liés à l'expérience partagée de l'exil. Ils ne vivent pas dans le mythe du retour. Depuis qu'ils ont quitté leur pays, ils ont décidé de commencer une nouvelle vie. Miguel, 53 ans, en témoigne : "Mon père est décédé à New York; j'ai deux frères aux États-Unis, ils sont américains; j'ai un frère en Espagne, il est espagnol; ma mère aussi est en Espagne et a la nationalité espagnole; j'ai un frère en Suède qui a la nationalité suédoise ; et moi, je suis en France et j'ai la nationalité française. Nous avons tous décidé pour diverses raisons de ne plus retourner au Chili... Je n'ai jamais eu de nostalgie pour le Chili, j'ai une distance par rapport à ce pays... En tout cas, je serai toute ma vie chilien, demain je serai moins chilien qu'hier, plus français qu'hier, mais je ne cesserai jamais d'être chilien et je ne serai jamais complètement français. Au début, cétait désagréable; maintenant je le vois comme une richesse..." Ce cas permet de mettre en évidence que la perte de liens forts au Chili et le questionnement politique sur ce qui s'est passé génèrent un désintérêt pour ce pays. Cela ne suppose pas pour autant une coupure totale avec les origines, car la moitié des contacts de ces exilés sont des compatriotes résidant en France. Dans le cas de Miguel, si cette appartenance hybride était au départ non désirée, elle est devenue un capital qu'il a appris à exploiter.

\section{Conclusion}

Les exilés politiques chiliens, accueillis en France comme des "héros", ont été perçus par les partisans de Pinochet comme des "ennemis" et par la gauche restée au Chili comme des personnes vivant un "exil doré". Peu à peu, ils sont devenus des migrants ordinaires. Certes, ils continuent à s'identifier comme des exilés politiques - condition subjective qui semble ne jamais pouvoir s'effacer. Chez eux, la mémoire reste à vif, mais cela ne se traduit pas par un combat politique. Le levier de leur départ obligé n'existe plus, et leurs appartenances, tout autant que leurs manières de vivre se confondent avec celles des autres migrants.

Comment ce glissement s'est-il opéré au cours de l'histoire, de leur histoire personnelle, qui est aussi l'histoire de leur pays ? Est-ce que l'installation de la dictature sur le long terme a affaibli le combat politique? Le facteur temps a certainement joué, mais il faudrait chercher les réponses en comprenant ce qui s'est passé au cours de cette période-là. Pendant les dix-sept ans de dictature, les divisions politiques de la gauche chilienne ont été aussi ressenties chez les exilés politiques en France, ce qui a eu une répercussion sur leur difficulté à construire une alternative politique pour le Chili. Plusieurs d'entre eux ont interrogé leurs propres convictions politiques. La longue durée de la dictature a généré une sorte de "normalisation" de la situation, qui s'est traduite en France par un affaiblissement progressif du soutien des partis politiques de la gauche française face à la cause chilienne. Après la difficile épreuve de l'exil, ils sont arrivés à l'accepter et à trouver une stabilité en France, ils ont vécu une recomposition psychologique, culturelle, socio-économique et relationnelle. L'abandon du mythe de retour est massif, rentrer est perçu comme un nouveau recommencement. Ils ont déjà reconstruit leur "chez soi". La situation des migrants en général, partagés entre deux sociétés et deux espaces géographiques différents (d'origine et d'accueil), se résout non pas par le passage d'un lieu à un autre ou par le remplacement d'un pays par un autre, mais par l'invention d'un troisième espace constitué par l'union des deux. Il en résulte une "sociabilité à la chilienne", avec notamment des liens forts et nombreux avec des compatriotes résidant en France.

Vingt-trois ans après le retour de la démocratie au Chili, ces exilés vivent une sorte de désenchante- 
ment car le pays qu'ils ont quitté a changé. La dictature a réussi à anéantir la résistance et à modeler une pensée et une société fondées sur la consommation, forme de vie que les exilés ne partagent pas toujours. L’absence de justice - malgré certains procès et condamnations - et le fort héritage de l'ancien régime après le rétablissement de la démocratie contribuent également à ce découragement. Au Chili, la période d'effervescence politique a été suivie d'une dépolitisation de la société. Plusieurs exilés politiques ont vécu un processus similaire. Cette situation commence à être ébranlée par la nouvelle génération, qui réclame un changement du système éducatif hérité de l'ère Pinochet. Les post-exilés politiques soutiennent ces demandes, parmi d'autres, lesquelles sont aussi partagées par des migrants non liés à l'exil politique. Ces revendications s'étendent de la communauté des Chiliens réfugiés politiques à celle des Chiliens dans son ensemble. 